\title{
Olfactory receptor phylogeny reveals conserved channels for sex pheromone and host plant signals in tortricid moths
}

\author{
Francisco Gonzalez ${ }^{1}$, Felipe Borrero-Echeverry ${ }^{1}$, Julia Josvai ${ }^{2}$, Maria Strandh ${ }^{3}$, Rikard \\ Unelius $^{4}$, Miklos Toth ${ }^{2}$, Peter Witzgall ${ }^{5}$, Marie Bengtsson ${ }^{5}$, and William Walker ${ }^{5}$ \\ ${ }^{1}$ Affiliation not available \\ ${ }^{2}$ Plant Protection Institute CAR HAS, Budapest, Hungary \\ ${ }^{3}$ Molecular Ecology and Evolution Lab, Dept. Biology, Lund University \\ ${ }^{4}$ Faculty of Health and Life Sciences, Linnaeus University, Kalmar \\ ${ }^{5}$ Swedish University of Agricultural Sciences
}

May 5, 2020

\begin{abstract}
The search for mates and food is mediated by volatile chemicals. Insects sense food odorants and sex pheromones through odorant receptors (ORs) and pheromone receptors (PRs), which are expressed in olfactory sensory neurons. Investigating the receptive range of these receptors instructs the identification of behaviourally relevant chemicals. Studying orthologous receptors and their ligands across taxa affords insights into the role of chemical communication in reproductive isolation and phylogenetic divergence. The female sex pheromone of green budworm moth Hedya nubiferana (Lepidoptera, Totricidae) is a blend of two unsaturated acetates, only a blend of both elicits male attraction. Females also produce codlemone, which is the sex pheromone of another tortricid, codling moth Cydia pomonella. Codlemone also attracts green budworm moth males. Concomitantly, green budworm and codling moth males are attracted to the food plant volatile pear ester. A congruent behavioural response to the same pheromone and plant volatile in two tortricid species suggests co-occurrence of dedicated odorant receptors. In codling moth, one PR is tuned to both compounds, the sex pheromone codlemone and the plant volatile pear ester. Our phylogenetic analysis finds that green budworm moth expresses an orthologous PR gene. Shared ancestry, and high levels of amino acid identity and sequence similarity, in codling and green budworm moth PRs offers an explanation for parallel attraction of both species to the same compounds. A conserved olfactory channel for a sex pheromone and host plant volatile substantiates the alliance of social and habitat signals in insect chemical communication. Field attraction assays confirm that in silico investigations of odorant receptors afford powerful predictions for an efficient identification of behaviour-modifying semiochemicals, for an improved understanding of the mechanisms of host plant attraction in insect herbivores and for the further development of sustainable insect control.
\end{abstract}

\section{Key words}

behaviour-modifying chemicals, reproductive behaviour, sustainable insect control, semiochemical, kairomone, olfaction, Tortricidae, Lepidoptera

\section{Introduction}

Olfactory perception of food cues and sex signals is intimately interconnected in insects (Reddy and Guerrero 2004; Varela et al.2011; Rouyar et al. 2015; Lebreton et al. 2017; Borrero-Echeverry et al. 2018; Conchou 
et al. 2019). Deciphering the chemicals encoding food and mates is basic to understanding insect ecology and evolution. Moreover, the knowledge of such behaviour-modifying chemicals can be applied for detection and environmentally safe control of insects (Ridgway et al. 1990; Witzgall et al. 2008, 2010a; Reddy and Guerrero 2010; Suckling et al. 2014; Evenden and Silk 2016; Gregg et al. 2018).

New tools for insect management are needed in the wake of a changing climate that accelerates insect invasions and outbreaks, aggravating food insecurity (Deutsch et al. 2018). Recent efforts to deregulate the most toxic compounds has left growers with few efficient insecticides (Chandler et al. 2011; Jactel et al. 2019). The overwhelming majority of insect species, however, does not feed on human food crops. Including pollination services, insects are integral to all terrestrial food webs. The overuse of synthetic pesticides affects non-target and beneficial insects and other arthropods, and is a contributing cause of the biodiversity apocalypse. This has been a point of debate since DDT (Carson 1962) and nonethless, evidence is accumulating for severe side effects of the currently most widely used family of insecticides, the neonicotinoids (Seibold et al. 2019; Yamamuro et al. 2019; Chmiel et al. 2019; Longing et al. 2020; Wagner 2020).

The establishment of pheromones and other semiochemicals as a species-specific and environmentally safe alternative to conventional insecticides has therefore always been an outstanding rationale for chemical ecology research. Air-permation with synthetic pheromone, for disruption of premating sexual communication, is used against a few key orchard and forest insects (Reddy and Guerrero 2010; Witzgall et al. 2010; Evenden and Silk 2015). Pheromone lures for specific and sensitive detection are available for hundreds of species. Such lures, in combination with traps, insect pathogens or insecticides, may even achieve population control, when the female sex becomes attracted (Ridgway et al. 1990; El-Sayed et al. 2009; Sucklinget al. 2014). In stark contrast to pheromones attracting insects for mating, only few semiochemicals have been identified that attract gravid females for oviposition. Designing female or bisexual lures is therefore a current main challenge towards a more widespread use of behaviour-modifying chemicals for insect control.

Identification of many hundreds of sex pheromones, across all insect orders (El-Sayed et al. 2016), has been facilitated by a mutual coordination of production and response in both sexes. Pheromones are produced in dedicated glands, produce strong antennal responses and immediately trigger a sequence of distinctive behaviours.

Identification of semiochemicals, or kairomones, that mediate oviposition behaviour meets substantial methodological difficulties. Synthetic plant volatile blends that have been found to attract insect herbivores typically build on compounds found across many plant species (Najar-Rodriguez et al. 2010; Tasin et al. 2010; Bruce and Pickett 2011; Lu et al. 2015). The attractant power of such ubiquitous plant volatiles is sometimes faint, compared with sex pheromones.

In comparison, plant compounds that are unique or characteristic for larval food plants have been found to mediate significant attractancy. One such key host plant compound is ethyl (E,Z)-2,4-decadienoate, pear ester, a bisexual attractant for codling moth Cydia pomonella(Lepidoptera, Tortricidae) (Light et al. 2001; Light and Knight 2005). Pear ester is efficient for population monitoring (Knightet al . 2013, 2019) and for behavioural disruption of codling moth larvae and adults, alone or combined with sex pheromone (Light and Knight 2011; Knight et al. 2012; Light and Beck 2012; Knight and Light 2013). The discovery of pear ester demonstrates the potential of kairomones to both improve pheromone-based techniques and to design stand-alone applications. That pear ester is released only in trace amounts from green apples (Gonzalez et al. 2020) underlines that the abundance of volatiles in plant headspace does not correlate with their behavioural saliency. Compounds released in large amounts often stem from main biosynthetic pathways shared by many plants, and cannot encode specific host plant finding.

The most widely employed tool for studying plant compounds mediating host attraction is gas chromatography coupled to electroantennographic detection. GC-EAD measures the response of the entire antenna to odorants (Arn et al. 1975), and biases compounds occurring in large amounts in headspace collections. GC-EAD was designed as an efficient and reliable tool for detection and identification of trace amounts of sex pheromones. GC-EAD suffers, however, from serious bias and produces false positives when screening 
plant headspace. Ubiquitous compounds present in large amounts, for example short aliphatic acetates or alcohols, farnesenes, linalools and caryophyllenes, invariably elicit an antennal response, generated by the ensemble of olfactory sensory neurons (OSNs) on the antenna, expressing the entire olfactory receptor (OR) repertoire. Typically, ORs respond to large amounts of volatiles that are structurally similar to their cognate ligands. A diffuse signal from many OSNs on the antenna is sufficient to produce electroantennograms, but its behavioural relevance is uncertain. An active compound such as pear ester, on the other hand, has been overlooked in GC-EAD recordings due to its low abundance.

The discovery of the genetic code of insect ORs (Clyne et al. 1999) enables a new approach. The ligand binding specificity of ORs determines the spectrum of volatile chemicals transmitted by OSNs from the antenna to olfactory centers in the brain. Sequencing antennal RNA extracts and annotation provides OR expression data and a first functional differentiation, between pheromone receptors (PRs) and ordinary ORs, responding to environmental odorants. Subsequent phylogenetic analysis groups orthologous ORs from related species and provides leads on putative ligands, through comparison with an accumulating database of deorphaned insect ORs (Fleischer et al. 2018, Robertson 2019). Single ORs are accordingly a tool of choice to interrogate the plant odorscape for bioactive compounds. A powerful experimental approach is to express ORs singly in defined sensilla of the antenna of Drosophila melanogaster (Dobritsa et al. 2003; Hallem et al. 2004), where they can be addressed with single sensillum electrophysiological recordings, coupled to gas chromatography (GC-SSR).

In codling moth Cydia pomonella (Lepidoptera, Tortricidae), Cpom OR3 has been deorphaned, following transcriptome analysis (Bengtsson et al. 2012, Walker et al. 2016) and heterologous expression (Bengtsson et al. 2014, Cattaneo et al. 2017, Wan et al. 2019). The main ligand of CpomOR3, which belongs to the PR clade, is the plant volatile pear ester (Light et al. 2001; Light and Knight 2005, Bengtsson et al. 2014). A recent assembly of the codling moth genome reveals presence of two copies of CpomOR3, which, according to functional characterization in Xenopus oocytes, respond to a lesser extent also to codling moth sex pheromone, codlemone (Wan et al. 2019). A seemingly conserved response in a closely related species underscores this deeply rooted interconnection of pheromone and plant volatiles. Green budworm moth Hedya nubiferana (Lepidoptera, Tortricidae) is attracted to codlemone (Arn et al. 1974, El-Sayed, 2019) and to pear ester (Schmidt et al. 2007, Jósvai et al. 2016).

We have investigated the response of green budworm moth $H$.nubiferana to codling moth sex pheromone and to pear ester, in laboratory and field bioassays. Comparative phylogenetic analysis of ORs in the antennal transcriptome of green budworm and codling moth confirm the behavioural evidence and suggest the presence of a conserved olfactory channel dedicated to these compounds, in both species. This demonstrates how functional characterization of ORs in model species such as codling moth (Bengtsson et al. 2014; Gonzalez et al. 2016), followed by in silico studies of antennal transcriptomes in the taxonomically related species will advance the identification of insect kairomones, and the development of insect management.

\section{Materials and methods}

\section{Insects}

Green budworm moth Hedya nubiferana Haworth (dimidioalbaRetzius) (Lepidoptera, Tortricidae) (Figure 1) is a polyphagous leafroller on Rosacean trees and shrubs and co-occurs with codling moth Cydia pomonella on apple, throughout the Northern hemisphere. The larvae feed on fruit in autumn and on flower buds in the spring (Bradley et al. 1979).

For pheromone analysis, last-instar larvae were field-collected in apple orchards in Scania (Sweden) during May. Larvae were fed with apple leaves and a semisynthetic agar-based diet (Rauscher et al. 1984). Pupae and adults were kept under a 18:6 h light-dark cycle in screen cages and were supplied with fresh apple branches and sucrose solution. For transcriptomic studies, H. nubiferana males were captured in pheromone 
traps baited with a 10:1:5-blend of ( $E, E$ )-8,10-dodecadienyl acetate ( $E$ 8,E 10-12Ac), ( $E$ )-8-dodecenyl acetate $(E$ 8-12Ac) and $Z$ 8-12Ac. Live males were taken to the laboratory and used for antennal dissection.

\section{Pheromone gland extraction and chemical analysis}

Female abdominal sex pheromone glands were dissected at the onset of the calling period, towards the end of the scotophase. Glands of 2- to 4-d old females were extracted in batches of 5 to 15 in $7 \mu \mathrm{L}$ of redistilled hexane for 1 min (Bäckman et al. 1997). Identification of female gland compounds by coupled gas chromatography-mass spectrometry (GC-MS) was done on a Hewlett Packard 5970 B instrument, with electron impact ionization $(70 \mathrm{eV})$, interfaced with a Hewlett Packard 5890 GC. Helium was used as carrier gas on a $30 \mathrm{~m} \times 0.25 \mathrm{~mm}$ DB-Wax column (J\&W Scientific, Folsom, CA, USA), programmed from $80^{\circ} \mathrm{C}$ (hold $2 \mathrm{~min}$ ) at $10^{\circ} \mathrm{C} / \mathrm{min}$ to $230^{\circ} \mathrm{C}$. The compounds were identified by comparing retention times and mass spectra of natural and synthetic compounds. Double bond position was determined by co-injection with synthetic samples and by evaluation of mass spectra.

\section{Field trapping}

The geometric isomers of $E$ 8,E 10-12Ac and $E$ 8,E 10-12OH were synthesized (Witzgall et al . 1993). All other compounds were purchased from S. Voerman (Institute for Pesticide Research, Wageningen, The Netherlands). Purity of synthetic pheromone compounds was [?] $96.2 \%$ (chemical) and [?] $99.7 \%$ (isomeric). Compounds in hexanic solution were formulated on red rubber septa (Merck ABS, Dietikon, Switzerland), which were replaced every 2 weeks. Tetra traps (Arn et al. 1979) were hung in apple trees at eye level, and were $c a .5 \mathrm{~m}$ apart within one replicate. Traps were placed in untreated apple orchards at Alnarp, Scania (Sweden) and at Halasztelek, Pest county (Hungary) and checked twice a week.

Further traps were placed in orchards treated with commercial pheromone dispensers for mating disruption of codling moth. These dispensers were polyethylene tubes containing $87 \mathrm{mg} E$ 8,E 10-12OH, $49 \mathrm{mg} 12 \mathrm{OH}$ and $10 \mathrm{mg}$ 14OH (Shin-Etsu Chemical Co., Tokyo), they were applied at a rate of 1000/ha.

For statistical analysis, trap captures were transformed to $\log (\mathrm{x}+1)$ and submitted to a 2-way ANOVA, followed by Tukey's test.

\section{Wind tunnel}

The wind tunnel had a flight section of $63 \times 90 \times 200 \mathrm{~cm}$ (Witzgall et al. 2001). Air was blown by a horizontal fan onto an array of activated charcoal cylinders. The wind tunnel was lit diffusely from above at 6 lux, the wind speed was $30 \mathrm{~cm} / \mathrm{s}$, and the temperature ranged from 22 to $24 \mathrm{degC}$. Two-day-old males were transferred to glass tubes $(2.5 \times 12.5 \mathrm{~cm})$ stoppered with gauze before testing. Males were flown individually, in batches of 15 , to one test stimulus. Two batches of 15 males were tested on one day, 1 to $3 \mathrm{~h}$ after onset of the, each blend was tested four times ( $\mathrm{n}=60$ males), on different days. The following types of behaviour were recorded: taking flight, flying upwind over $100 \mathrm{~cm}$ towards the source, and landing at the source.

\section{Dissection of antennae and RNA extraction}

Antennae of 100 adult males were dissected with forceps and transferred into a 1.5-mL microcentrifuge tube (Eppendorf, Hamburg, Germany) held in liquid nitrogen. Thereafter, $500 \mu \mathrm{L}$ of Trizol were added to the excised antennae.

Total RNA was extracted and purified following Trizol-based extraction protocol and spin column purification with the RNeasy Mini Kit (Qiagen, Venlo, The Netherlands). Briefly, antennae held in the Eppendorf tube with Trizol were manually homogenized with a pestle. The tube was placed in liquid nitrogen and then allowed to thaw at room temperature. The sample was then homogenized again with a pestle and another $500 \mu \mathrm{L}$ of Trizol were added to the tube. The tube was vortexed and incubated at room temperature for 5 
min, $200 \mu \mathrm{L}$ of chloroform (Riedel de Haen, Seelze, Germany) was added to the sample and the tube was vortexed again for $20 \mathrm{~s}$ and incubated at room temperature for $15 \mathrm{~min}$. Samples were centrifuged at $4^{\circ} \mathrm{C}$ for $15 \mathrm{~min}$ at maximum speed. The aqueous upper phase was transferred to a clean 1.5-mL centrifuge tube and an equal amount of $100 \%$ isopropanol (Sigma Aldrich, Saint Louis, MO, USA) was added along with $3 \mu \mathrm{L}$ of $5 \mathrm{mg} / \mathrm{mL}$ of glycogen (Life Technologies, Carlsbad, Ca, USA). Samples were mixed by inversion a couple of times and stored at $-20^{\circ} \mathrm{C}$ overnight.

The next day, the sample was centrifuged at $4^{\circ} \mathrm{C}$ for $15 \mathrm{~min}$ at maximum speed. The supernatant was decanted and the excess of liquid extracted with a pipette without disturbing the pellet, $1 \mathrm{~mL}$ of cold $70 \%$ ethanol was added to the pellet sample and centrifuged at $4^{\circ} \mathrm{C}$ for $10 \mathrm{~min}$ at $7500 \mathrm{RCF}$. Supernatant was discarded and $100 \mu \mathrm{L}$ of RNAse free water (Life Technologies, Carlsbad, Ca, USA) was added to the tube. Extracted RNA was then purified with the RNeasy Mini Kit (Qiagen, Venlo, The Netherlands); $350 \mu \mathrm{L}$ of Buffer RLT and $250 \mu \mathrm{L}$ of $100 \%$ ethanol were added to the sample. The sample was transferred to RNeasy spin columns and the RNA was fixed to the filter membrane via centrifugation at room temperature for $15 \mathrm{~s}$ at $10000 \mathrm{RCF}$. According to manufacturers recommendation, RNA purification was completed with the RNA Cleanup Protocolol, including an on-column DNase digestion, performed with the RNase Free DNase system (Qiagen). Total RNA was quantified with a Nanodrop 1000 spectrophotometer (Thermo Fisher Scientific, Waltham, MA, USA).

\section{RNA sequencing and bioinformatics}

RNA sequencing at the National Genomics Infrastructure (NGI, Uppsala, Sweden) followed the standard protocols for Illumina Sequencing (Illumina, CA, USA), sequence read files were sent to UPPMAX Computational Science Server (Uppsala, Sweden). Two .fq files were produced, one containing all left-pair reads and another containing all right-pair reads.

The .fq files were used as a starting point to assemble the transcriptome, annotate the genes and calculate their expression (see Walker et al. 2016). Quality control analysis was performed using the software Trimmomatic (version 0.32), and all reads with a PHRED score lower than 20 were removed. Processed reads were then assembled, de novo, into one transcriptome using Trinity (version r2014717; Grabherr et al. 2011). Cd-hit-est (version 4.5.4-2011-03-07), was used to identify and remove redundant sequences that share $98 \%$ or greater identity with other sequences ( $\mathrm{Li}$ and Godzik 2006). The processed transcriptome was used to compare and annotate gene transcripts according to their homology to protein sequences of $C$. pomonella (Walker et al. 2016), using blast (version 2.2.29). Top blast hit transcript clusters with similarity to putative pheromone receptors of $C$. pomonella were extracted and translated into protein sequence with the ExPASY web translate tool (Artimo et al. 2012). Translated sequences with open reading fragments (ORFs) shorter than $50 \%$ of the average length of a OR (428 amino acids) were excluded from analysis. Sequences were aligned to putative PRs from C. pomonella (Walker et al. 2016) and all new putative PRs from $H$. nubiferana were named according to the closest homolog of C. pomonella .

To estimate the expression of these putative PRs in the antennae the RSEM software package (version 1.2.12; $\mathrm{Li}$ and Dewey 2013), including Bowtie (version 0.12.6; Langmead et al. 2009) and Samtools (version 0.1.19; Li et al. 2009) were used, allowing measurement of transcript abundance estimates as fragments per kilobase of transcript per million mapped reads (FPKM) (Trapnell 2010).

\section{Phylogenetic analysis}

Sequences of predicted pheromone receptors from C. pomonella(Walker et al. 2016), Epiphyas postvittana (Corcoran et al. 2015), Grapholita molesta (Li et al. 2015) and Bombyx mori(Krieger et al. 2005), were used for comparison with putative PRs ofH. nubiferana. All amino acid sequences were aligned using MAFFT online (version 7.220; http://mafft.cbrc.jp/alignment/server/phylogeny.html) with the FFT-NS-i iterative refinement method, with JTT200 scoring matrix, and default parameters. Aligned sequences were used to calculate the best fitting model for comparison in MEGA6 software (Tamura et al. 2013). Then, 
a Maximum Likelihood Tree was constructed using the JTT $+\mathrm{F}+\mathrm{G}$ model with bootstrap support inferred from 500 replicates.

\section{Results}

\section{Sex pheromone identification}

Analysis of green budworm moth $H$. nubiferana pheromone gland extracts by GC and GC-MS showed eight further compounds, in addition to the previously identified acetates (Frerot et al. 1979). The major compound $E$ 8,E 10-12Ac was accompanied by the monounsaturated 8- and 10-dodecenyl acetates, its three geometric isomers ( $E Z, Z E$, and $Z 8, Z 10-12 \mathrm{Ac}$ ) as well as the analogous alcohol codlemone, $E 8, E 10-12 \mathrm{OH}$ (Table 1).

Field attraction of $H$. nubiferana males to compounds identified from the female gland confirms that the sex pheromone of $H$. nubiferana is a blend of $E$ 8,E 10-12Ac and $Z$ 8-12Ac (Table 2; Frerot et al. 1979). The main compound, $E$ 8,E 10-12Ac by itself was not attractive, while addition of $Z$ 8-12Ac had a strong synergistic effect $(\mathrm{F}(7,72)=61.95, P<0.0001)$. Addition of $E$ 8-12Ac further increased male attraction in untreated apple orchards, but the difference was not significant. Blends of $E$ 8,E 10-12Ac and the [?] 10-12 monoenes or the analogous alcohol, codlemone, did not produce significant captures. Adding these compounds to the 3-component acetate blend slightly diminished trap catch (Table 2).

The gland compounds identified from female glands with no apparent effect on attraction may be biosynthetic by-products or precursors. A study of the female effluvium will show whether they are released at all, and at which ratio. The full blend of compounds may also carry information that cannot be revealed by a field trapping test.

\section{Attraction to codlemone and pear ester}

A trap test in an apple orchard adjacent to a pea field corroborates that codlemone acetate $E$ 8,E $10-12 \mathrm{Ac}$ as a single compound does not attract green budworm moth. Attraction of pea moth confirms that the trap lures released $E$ 8,E 10-12Ac at high isomeric purity (Table 3; Witzgall et al. 1993, 1996). In comparison, traps baited with codlemone alone regularly captured few green budworm moth males, in addition to codling moth. Blends of codlemone and codlemone acetate attract far fewer codling moths and no green budworm moths at all (Table 3).

Interestingly, a blend of codlemone and its three geometric isomers significantly increased green budworm moth captures over codlemone alone (Table $4 ; \mathrm{F}(7,72)=2.62, P=0.04413)$. In contrast, this isomer blend captured fewer codling moth males (Table $4 ; \mathrm{F}(7,72)=4.22, P=0.02135$; El-Sayed et al . 1998).

Green budworm moth has also been reported to respond to pear ester (Schmidt et al. 2007; Jósvai et al. 2016). A further field test in Hungary confirmed this and showed that addition of codlemone to pear ester does not enhance attraction of either sex (Table 5).

Orchard mating disruption treatments with codlemone strongly diminished attraction of $H$. nubiferana males to pheromone traps (Table 2), corroborating a behavioural effect of codlemone via a dedicated olfactory channel. This supports the idea that communication disruption in moths may be achieved with single pheromonal compounds or incomplete pheromone blends (Cardé and Minks 1995; Porcel et al. 2015), which is of practical importance for the implementation of pheromonal control of codling moth and leafrollers in European orchards. 


\section{Phylogenetic analysis and antennal expression}

Hedya nubiferana Haworth and Hedya dimidioalba Retzius are synonymous taxonomic names for green budworm moth. The National Center for Biotechnology Information (NCBI) lists OR sequences (including PRs) as "HnubOR\#\#".

Predicted putative PRs from $H$. nubiferana clustered in 4 different subfamily clades when compared with PRs from other tortricid species (Figure 2A). Several of these displayed homology to receptors in Cydia pomonella (CpomOR3, CpomOR6 and CpomOR22) and these relationships were supported by boostrap values above 95.

Notably, HnubOR6 was $>50 \%$ similar to CpomOR6. Sequence comparison analysis revealed that CpomOR1 and HnubOR2 shared $49 \%$ amino acid identity and $66 \%$ similarity, while the OR3 orthologs of both species shared $64 \%$ and $76 \%$ identity and similarity, respectively. Amino acid differences between these putative PRs are observed across the entire length of the protein sequences (Figure 3 ).

Abundance estimation of the predicted sequences showed that the most highly expressed were HnubOR6 and HnubOR2. The other 3 putative PRs detected in male antennae were one or two orders of magnitude lower (Figure 2B).

\section{Discussion}

\section{Green budworm moth response to codlemone and pear ester}

The empirical finding that green budworm moth $H$. nubiferana males respond to codling moth $C$. pomonella sex pheromone and kairomone, codlemone and pear ester, correlates with the antennal transcriptomes of these two species. Molecular phylogenetics of olfactory receptors, informed by behavioural and functional data, generates sound hypotheses for the identification of semiochemicals driving olfactory behaviour.

Food and mate finding, the essential components of insect reproductive behaviour, depend on a finite number of ORs encoding relevant odour signals. Peripheral olfactory perception employs 39 ORs in the fruit flyDrosophila melanogaster (Menuz et al. 2014, Grabe et al. 2015), 58 ORs in codling moth $C$. pomonella (Walker et al. 2016) and a similar number of ORs has been found in other tortricids (Corcoran et al. 2015, Steinwender et al. 2015, Rochas et al. 2018). Insect OR genes are under strong selection (McBride and Arguello 2007, Sanchez-Gracia et al. 2009; Arguello et al. 2016, Robertson 2019) and orthologous receptors descending from shared ancestral genes, which are conserved across taxonomic clades, such as CpomOR3 and HnubOR3 (Figure 2), likely play adapative roles.

Functional characterization of CpomOR3, a codling moth OR, has established pear ester as its principal ligand. This was achieved through heterologous expression of CpomOR3 in olfactory sensory neurons of ab3 and T1 antennal sensilla in Drosophila melanogaster, followed by single sensillum electrophysiological recordings (SSR) (Bengtsson et al. 2014; Gonzalez et al. 2016) and has meanwhile been corroborated by luminescence assays after expression in human embryonic kidney cells and Xenopus oocytes (Cattaneo et al. 2017, Wan et al. 2019). CpomOR3, albeit tuned to a plant volaile compound, is part of the lepidopteran pheromone receptor (PR) clade (Bengtsson et al. 2012, 2014; Walker et al. 2016).

The hypothesis that $H$. nubiferana perceives pear ester via HnubOR3 is parsimonious: a PR phylogeny of tortricid moths (Figure 2A), together with sequence similarity analysis (Figure 3) show that CpomOR3 and HnubOR3 are close. In addition, HnubOR3 and CpomOR3 are among the most abundant transcripts in the male antenna (Figure 2B; Walker et al. 2016). This compares to the receptor orthologs CpomOR19 and SlitOR19 (Spodoptera littoralis ). Following functional characterization of SlitOR19, ligand affinity of CpomOR19 was predicted on the basis of amino acid sequence similarity (Gonzalez et al. 2015). 
Oriental fruit moth Grapholita molesta, although taxonomically closer to $C$. pomonella than to $H$. nubifera$n a$ (Bradley et al. 1979; Regier et al. 2012), is not known to respond to dienic pheromone compounds or pear ester, which is corroborated by our PR phylogeny (Figure 2). The broad host range of G. molestaoverlaps only partially with $C$. pomonella and $H$.nubiferana food plants.

\section{Attraction to sex pheromone and codlemone employs distinct olfactory channels}

Attraction of green budworm moth $H$. nubiferana to its multicomponent sex pheromone and to codling moth pheromone employs separate olfactory channels. Codlemone $E$ 8,E 10-12OH does not mimic the $H$. nubiferana main pheromone compound codlemone acetate $E$ 8,E 10-12Ac, since codlemone is active as single compound, while codlemone acetate is not (Tables 2,3). Tortricid moths differentiate analogous alcohol from acetate pheromone compounds at high resolution (Witzgall et al. 1991, 1993, 1996, 2010b), probably due to steric differences of the functional groups (Bengtsson et al. 1990). PR phylogeny, together with expression levels in H. nubiferana and C. pomonella (Figure 2; Walker et al. 2016), suggests that CpomOR1 and HnubOR2 are tuned to codlemone, and CpomOR6 and HnubOR6 to codlemone acetate (Catteaneo et al. 2016). In codling moth, codlemone acetate is a pheromone synergist or antagonist, when added to the main pheromone compound codlemone at small and large amounts, respectively (Hathaway et al. 1974; Witzgall et al. 2001).

Presence of two pheromone channels in H. nubiferana males is reminiscent of the "hopeful monster" (Baker 2002, Dietrich 2003) and "asymmetric tracking" (Phelan 1992) concepts, suggesting that new communication channels arise through saltational shifts in female pheromone production, which are subsequently tracked by the male sex. Such shifts are facilitated by redundancies in the PR repertoire.

Three related species, $H$. ochroleucana, $H$. prunianaand $H$. salicella are best attracted to the $Z$,Eisomers of codlemone and codlemone acetate, and $Z, E$-codlemone is active in codling moth (El-Sayed et al. 1998; Witzgall et al. 2010b). A candidate PR for $Z, E$-codlemone is HnubOR1 (Figure 2). Regarding HnubOR7a and HnubOR7b, which are close to GmolOR1 and GmolOR11, we hypothesize that they respond to the minor acetate pheromone components $(Z)$ - and (E )-8-dodecenyl acetate (Tables 2,3), which are main pheromone compounds of Oriental fruit moth G. molesta (Carde et al. 1979).

\section{In silico identification of semiochemicals for the development of insect control}

Semiochemicals are efficient tools for insect control, by air-permeation and mass trapping (El-Sayed et al. 2009; Witzgall et al. 2010a). The know-how of behaviour-modifying chemicals can also be brought to application through push-pull techniques or plants with modified metabolite release profile (Khan et al. 2014; Stenberg et al. 2015; Tamiru et al. 2015). A current bottleneck and urgent research challenge for further advancement is our understanding of which plant volatile metabolites mediate host recognition in phytophagous insects.

Availability of plant volatiles which attract insects to mating sites, elicit oviposition or feeding in adults and larvae leads to multiple applications. Pear ester, for example, is efficient for monitoring codling moth males and females, it can be used to supplement pheromone-based communication disruption and is a stand-alone tool for disruption of larval host-finding and feeding (Schmidt et al. 2008; Light and Knight 2011, Light and Beck 2012; Knight et al. 2012, Knight and Light 2013, Kovanci 2015, Light 2016). Another example is an efficient kairomone lure for apple fruit moth Argyresthia conjugella, based on characteristic host plant compounds (Bengtsson et al. 2006; Knudsen and Tasin 2015; Knudsen et al. 2008, 2017).

Current research on behaviourally active plant metabolites relies on electroantennogram recordings, coupled to a gas chromatograph. The GC-EAD method was originally conceived for sex pheromone identification (Arn et al. 1975). The conundrum, when working with plant volatiles, is that antennal recordings do not provide information on behavioural activity. GC-EAD recordings are also biased by the most abundant compounds, which invariably produce an antennal response. Recordings from single sensilla provide a solution, but in vivo 
recordings from sensilla other than $s$. trichodea, containing pheromone-sensitive neurons, are delicate. In codling moth, SSR recordings produced conclusive results when investigating pheromones, not plant volatiles (Bäckman et al. 2000; Ansebo et al. 2005).

In silico identification of OR ligands now emerges as an opportune advancement for plant semiochemical research. Phylogenetic analysis of OR gene sequences, in combination with functional characterization of selected ORs in model species, affords powerful predictions about behaviour-modifying plant volatiles. Identification of ORs, following antennal RNA sequencing, and hypotheses concerning their putative ligands is facilitated by the rapidly accumulating database of insect ORs. Antennal transcriptomes highlight highly expressed or sex-specific ORs, which become prime research targets. Furthermore, heterologous expression of ORs in select sensory neurons inDrosophila enables single sensillum recordings as a convenient and reliable approach for unambiguous identification of OR ligands (Dobritsa et al. 2003; Hallem et al. 2004; Gonzalez et al. 2016).

Promising targets for future work include, for example, tephritid fruit flies, in view of our thorough knowledge of Drosophila ORs (Liuet al . 2016; Muench and Galizia 2016) or moths from several families, aided by a rapidly accumulating database of lepidopteran antennal transcriptomes (e.g. Zhang et al. 2013; Cao et al. 2014; Jiang et al. 2014; Zhang et al. 2014, 2017; Corcoran et al. 2015; Koenig et al. 2015; Li et al. 2015; Park et al. 2015; Steinwender et al. 2015; Zeng et al. 2015; Zhang et al. 2015; Dong et al. 2016; Jia et al. 2016, 2018; Chang et al. 2017; Feng et al. 2017; Yang et al. 2017; Du et al. 2018; Rochas et al. 2018; Tian et al. 2018)

\section{Interaction of plant volatiles and pheromones}

Green budworm moth attraction to pear ester and codlemone is intriguing, because it provides further evidence for the association of olfactory channels dedicated to social and environmental signals in phytophagous insects.

Transcriptome data and phylogenetic context confirm this association. CpomOR3 is tuned to the plant volatile pear ester, while it belongs to the pheromone receptor clade (Figure 2A, 3; Bengtsson et al . 2012, 2014; Walker et al . 2016). That PRs respond to pheromones and plant volatiles has even physiological consequences: OR genes with highest sequence similarity tend to be expressed in OSNs that project to neighbouring glomeruli in the antennal lobe, facilitating interactions between the circuits encoding these signals (Couto et al. 2005, Krieger et al. 2009, Ramdya and Benton 2010). This has indeed been confirmed in codling moth, by intracellular recordings from olfactory projection neurons and functional imaging of the antennal lobe, showing a powerful synergistic interaction between codlemone and pear ester (Trona et al. 2010, 2013).

HnubOR3 has not been deorphaned, but the recent discovery that CpomOR3 responds to pear ester and to a lesser extent also to codlemone (Wan et al. 2019) provides an explanation for consistent attraction of $H$ .nubiferana to codlemone (Tables 3, 4; Arn et al. 1974). Codling moth C. pomonella and H. nubiferana both feed on apple, but belong to different tortricid tribes (Bradley et al. 1979; Regier et al. 2012). Occurrence of conserved olfactory genes contributing to mate finding and host plant attraction lends further support to the concept that host plant recognition and sexual communication are interlinked (Borrero-Echeverry et al. 2018 ) and that a combination of natural and sexual selection gives rise to reproductive isolation in insect herbivores (Paterson 1978, Boughman 2002, Rosenthal 2017). A more complete analysis of olfactory genes and their behavioural and ecological functions will contribute to the study of phylogenetic divergence in phytophagous insects. Equally rewarding is the perspective that this research also drives the development of semiochemicals for efficient and sustainable insect control. 


\section{Acknowledgements}

This work was supported by Carl Tryggers Stiftelse för Vetenskaplig Forskning, Formas (project 2011-1370) and the Linnaeus environment "Insect Chemical Ecology, Ethology, and Evolution (IC-E3)" (Formas, SLU). The authors acknowledge support from Science for Life Laboratory, the National Genomics Infrastructure (NGI), and Uppmax for providing assistance in massive parallel sequencing and computational infrastructure. Two reviewers provided constructive and valiable comments.

\section{Author Contributions}

Transcriptome analysis by FG and FB-E, under supervision by WBW. Field tests in Sweden by JKJ and MS, under supervision by MT, MB and PW, including preparation of field lures. RU synthesized the geometric isomers of E8,E10-12Ac. Pheromone identification by MB and PW. All authors contributed to the ms, first draft by PW.

\section{Data archiving statement}

Transcriptome raw reads sequence data are available through the NCBI Sequence Read Archive (Accession Number: SRX1741573). Putative pheromone receptor sequences identified from the H. nubiferana transcriptome assembly are available through NCBI, and are included in a Transcriptome Shotgun Assembly project that has been deposited at DDBJ/EMBL/GenBank (accession numbers KY283585.1, KY283590.1 and KY283600.1). 


\section{Literature cited}

Ansebo, L., Ignell, R., Löfqvist, J. and Hansson, B. S. 2005. Responses to sex pheromone and plant odours by olfactory receptor neurons housed in sensilla auricillica of the codling moth, Cydia pomonella (Lepidoptera: Tortricidae). Journal of Insect Physiology 51:1066-1074. (doi:10.1016/j.jinsphys.2005.05.003)

Arguello JR, Cardoso-Moreira M, Grenier JK, Gottipati S, Clark AG, Benton R (2016). Extensive local adaptation within the chemosensory system following Drosophila melanogaster's global expansion. Nature Communications 7:11855. (doi:10.1038/ncomms11855)

Arn H, Schwarz C, Limacher H, Mani E (1974). Sex attractant inhibitors of the codling moth Laspeyresia pomonella L. Experientia 30:1142-1144. (doi:10.1007/BF01923655)

Arn H, Städler E, Rauscher S (1975). The electroantennographic detector - a selective and sensitive tool in the gas chromatographic analysis of insect pheromones. Zeitschrift für Naturforschung 30c:722-725. (doi:10.1515/znc-1975-11-1204)

Arn H, Rauscher S, Schmid A (1979). Sex attractant formulations and traps for the grape moth Eupoecilia ambiguella Hb. Mitteilungen der Schweizer Entomologischen Gesellschaft 52:49-55.

Artimo P, Jonnalagedda M, Arnold K, Baratin D, Csardi G, de Castro E, Duvaud S, Flegel V, Fortier A, Gasteiger E, Grosdidier A, Hernandez C, Ioannidis V, Kuznetsov D, Liechti R, Moretti S, Mostaguir K, Redaschi N, Rossier G, Xenarios I, Stockinger H (2012). ExPASy: SIB bioinformatics resource portal. Nucleic Acids Research 40:W597-W603. (doi:10.1093/nar/gks400)

Bäckman A-C, Bengtsson M, Witzgall P. 1997. Pheromone release by individual females of codling moth, Cydia pomonella L. (Lepidoptera: Tortricidae). Journal of Chemical Ecology 23:807-815. (doi:10.1023/B:JOEC.0000006412.16914.09)

Bäckman A-C, Anderson P, Bengtsson M, Löfqvist J, Unelius CR, Witzgall P (2000). Antennal response of codling moth males, Cydia pomonella (L.) (Lepidoptera: Tortricidae), to the geometric isomers of codlemone and codlemone acetate. Journal of Comparative Physiology A 186:513-519. (doi:10.1007/s003590000)

Baker TC (2002). Mechanism for saltational shifts in pheromone communication systems. Proceedings of the National Acadademy of Science USA 99:13368-13370. (doi:10.1073/pnas.222539799)

Bengtsson M, Liljefors T, Hansson BS, Löfstedt C, Copaja SV (1990). Structure-activity relationships for chain-shortened analogs of ( $Z$ )-5-decenyl acetate, a pheromone component of the turnip moth, Agrotis segetum . Journal of Chemical Ecology 16:667-684. (doi:10.1007/BF01016478)

Bengtsson JM, Trona F, Montagne N, Anfora G, Ignell R, Witzgall P, Jacquin-Joly E (2012). Putative chemosensory receptors of the codling moth, Cydia pomonella, identified by antennal transcriptome analysis. PLoS One 7(2):e31620. (doi:10.1371/journal.pone.0031620)

Bengtsson JM, Gonzalez F, Cattaneo AM, Montagne N, Walker WB, Bengtsson M, Anfora G, Ignell R, Jacquin-Joly E, Witzgall $P$ (2014). A predicted sex pheromone receptor of codling mothCydia pomonella detects the plant volatile pear ester. Front Ecol Evol 2:33 (doi:10.3389/fevo.2014.00033)

Bengtsson M, Jaastad G, Knudsen G, Kobro S, Bäckman A-C, Pettersson E, Witzgall P (2006). Plant volatiles mediate attraction to host and non-host plant in apple fruit moth,Argyresthia conjugella . Entomolgia Experimentalis et Applicata 118:77-85. (doi:10.1111/j.1570-7458.2006.00359.x)

Borrero-Echeverry F, Bengtsson M, Nakamuta K, Witzgall P (2018). Plant odour and sex pheromone are integral elements of specific mate recognition in an insect herbivore. Evolution 72:2225-2233. (doi:10.1111/evo.13571)

Boughman JW (2002). How sensory drive can promote speciation. Trends in Ecology and Evolution 17:571-577. (doi:10.1016/S0169-5347(02)02595-8)

Bradley JD, Tremewan WG, Smith A (1979). British tortricoid moths. Tortricidae: Olethreu- 


\begin{tabular}{llll}
\hline Compound & Short form & $\mathrm{ng} /$ female & $\%$ \\
Decyl actate & $10 \mathrm{Ac}$ & 0.2 & 2 \\
Dodecyl acetate & $12 \mathrm{Ac}$ & 1.0 & 15 \\
$(Z)$-5-dodecenyl acetate & $Z 5-12 \mathrm{Ac}$ & 0.1 & 2 \\
$(E)$-8-dodecenyl acetate & $E 8-12 \mathrm{Ac}$ & 0.7 & 10 \\
$(Z)$-8-dodecenyl acetate & $Z 8-12 \mathrm{Ac}$ & 3.6 & 56 \\
$(E)$-10-dodecenyl acetate & $E 10-12 \mathrm{Ac}$ & 0.7 & 11 \\
$(Z)$-10-dodecenyl acetate & $Z 10-12 \mathrm{Ac}$ & 0.1 & 2 \\
$(Z, E)$-8,10-dodecadienyl acetate & $Z 8, E 10-12 \mathrm{Ac}$ & 0.3 & 4 \\
$(E, E)-8,10$-dodecadienyl acetate & $E 8, E 10-12 \mathrm{Ac}$ & 6.5 & 100 \\
$(E, Z)-8,10$-dodecadienyl acetate & $E 8, Z 10-12 \mathrm{Ac}$ & 0.4 & 6 \\
$(Z, Z)-8,10$-dodecadienyl acetate & $Z 8, Z 10-12 \mathrm{Ac}$ & $<0.01$ & trace \\
$(E, E)-8,10$-dodecadienol & $E 8, E 10-12: \mathrm{OH}$ & 0.4 & 6 \\
\hline
\end{tabular}

Table 2 . Field attraction of $H$. nubiferanamales to components identified from the female pheromone gland, in untreated apple orchards $(\mathrm{N}=10)$ and orchards permeated with codlemone, $E$ 8, $E$ 10-12OH $(\mathrm{N}=6)$, June to July 1997. Means followed by the same letter are not significantly different (Tukey test, $\mathrm{F}(7,72)=61.95$, $P<0.0001)$.

\begin{tabular}{|c|c|c|c|c|c|c|c|c|}
\hline Compound & & & & $\mu \mathrm{g} /$ trap & & & & \\
\hline$E 8, E 10-12 \mathrm{OH}$ & 10 & 10 & 10 & 10 & 10 & 10 & 10 & 10 \\
\hline$E 8, Z 10-12 \mathrm{OH}$ & & 0.5 & 2 & & & & & 2 \\
\hline$Z 8, E 10-12 \mathrm{OH}$ & & & & 0.5 & 2 & & & 2 \\
\hline$Z 8, Z 10-12 \mathrm{OH}$ & & & & & & 0.5 & 2 & 2 \\
\hline H. nubiferana & $2.0 \mathrm{~b}$ & $3.5 \mathrm{ab}$ & $3.2 \mathrm{ab}$ & $2.3 \mathrm{ab}$ & $2.1 \mathrm{ab}$ & $1.8 \mathrm{~b}$ & $3.8 \mathrm{ab}$ & $6.6 \mathrm{a}$ \\
\hline C. pomonella & $8.0 \mathrm{a}$ & $9.0 \mathrm{a}$ & $4.8 \mathrm{ab}$ & $10.5 \mathrm{a}$ & $11.9 \mathrm{a}$ & $10.2 \mathrm{a}$ & $6.4 \mathrm{ab}$ & $3.2 \mathrm{~b}$ \\
\hline
\end{tabular}

Table 3 . Field trapping in apple orchards $(\mathrm{N}=10)$ and wind tunnel attraction $(\mathrm{N}=60)$ of $H$. nubiferana males to compounds identified from the female pheromone gland. Field traps attracted also codling moth $C$. pomonella and pea moth Cydia nigricana. Means followed by the same letter are not significantly different (Tukey test, $P<0.05$ ).

Compound

$E 8, E 10-12 \mathrm{Ac}$

$E 8-12 \mathrm{Ac}$

$Z 8-12 \mathrm{Ac}$

E8,E10-12OH

H. nubiferana

C. nigricana

C. pomonella

Taking flight

Upwind flight

Landing at source
10

$\begin{array}{ll}10 & 10 \\ & 1 \\ & 5\end{array}$

Number of males/trap

$0 \mathrm{c}$

$20.1 \mathrm{a}$

0

Male H. nubiferana wind tunnel behaviour (\%) $48 \mathrm{a}$

$0 \mathrm{~b}$

$0 \mathrm{~b}$
Number of males/trap

$57.5 \mathrm{a}$

$3.4 \mathrm{~b}$

0

Male H. nubiferana wind tunnel behaviour (\%)

$51 \mathrm{a}$

39 a

$22 \mathrm{a}$ $\mu \mathrm{g} /$

10

1

5

10

$\mathrm{Nu}$

53.

0

0

$\mathrm{Ma}$

47

33

* not tested 
Table 4 . Field trapping of $H$. nubiferana and C. pomonella males to the geometric isomers of codlemone $E$ $8, E 10-12 \mathrm{OH}(\mathrm{N}=10)$. Means followed by the same letter are not significantly different (Tukey test, $P$ $<0.05)$.

\begin{tabular}{lllllllll}
\hline Compound & & \multicolumn{7}{c}{$\mu \mathrm{g} / \mathrm{trap}$} \\
$E 8, E 10-12 \mathrm{OH}$ & 10 & 10 & 10 & 10 & 10 & 10 & 10 & 10 \\
$E 8, Z 10-12 \mathrm{OH}$ & & 0.5 & 2 & & & & & 2 \\
$Z 8, E 10-12 \mathrm{OH}$ & & & & 0.5 & 2 & & & 2 \\
$Z 8, Z 10-12 \mathrm{OH}$ & & & & & & 0.5 & 2 & 2 \\
H. nubiferana & $2.0 \mathrm{~b}$ & $3.5 \mathrm{ab}$ & $3.2 \mathrm{ab}$ & $2.3 \mathrm{ab}$ & $2.1 \mathrm{ab}$ & $1.8 \mathrm{~b}$ & $3.8 \mathrm{ab}$ & $6.6 \mathrm{a}$ \\
C. pomonella & $8.0 \mathrm{a}$ & $9.0 \mathrm{a}$ & $4.8 \mathrm{ab}$ & $10.5 \mathrm{a}$ & $11.9 \mathrm{a}$ & $10.2 \mathrm{a}$ & $6.4 \mathrm{ab}$ & $3.2 \mathrm{~b}$ \\
\hline
\end{tabular}

Table 5 . Field trapping of $H$. nubiferana males and females with blends of pear ester, ethyl (E,Z)-2,4decadienoate, and codlemone $E 8, E 10-12 \mathrm{OH}(\mathrm{N}=4)$. Means followed by the same letter are not significantly different (Tukey test, $P<0.05$ ).

\begin{tabular}{lllll}
\hline Compound & & $\mu \mathrm{g} /$ trap & \\
Pear ester & 6.000 & 6.000 & 6.000 & 6.000 \\
$E 8, E 10-12 \mathrm{OH}$ & & 1 & 3 & 10 \\
& Number of moths/trap & Number of moths/trap & Number of moths/trap & Number of moths/trap \\
Males & $0.3 \mathrm{a}$ & $0.3 \mathrm{a}$ & $0.1 \mathrm{a}$ & $0.1 \mathrm{a}$ \\
Females & $0.1 \mathrm{a}$ & $0.1 \mathrm{a}$ & $0.04 \mathrm{a}$ & $0.2 \mathrm{a}$ \\
\hline
\end{tabular}

\section{Figure legends}

Figure 1. Green budworm moth Hedya nubiferana Haworth (dimidioalba Retzius) (Lepidoptera, Tortricidae). Foto by Lubomír Hlásek.

Figure 2 . (A) Maximum likelihood unrooted phylogenetic tree of candidate Hedya nubiferana (Hnub) pheromone receptors (PR), including PR sequences from other tortricids, Cydia pomonella (Cpom), Epiphyias postvittana (Epos), Grapholita molesta (Gmol). Bombyx mori (Bmor) served as outgroup, node support was assessed with 500 bootstrap replicates, values $>70 \%$ are shown. (B) Hedya nubiferana PR transcript abundance estimates in male antennae. Expression levels quantified by RSEM, and indicated as fragments per kilobase of transcript per million reads (FPKM).

Figure 3. Amino acid alignments of selected Hedya nubiferana and Cydia pomonella PRs. HnubOR2 and CpomOR1 (A), HnubOR3 and CpomOR3 (B). Amino acid sequence differences are indicated as highly (:) and moderately (.) conservative, and as non-conservative substitutions (blanks). Asterisks indicate identity across both sequences. 


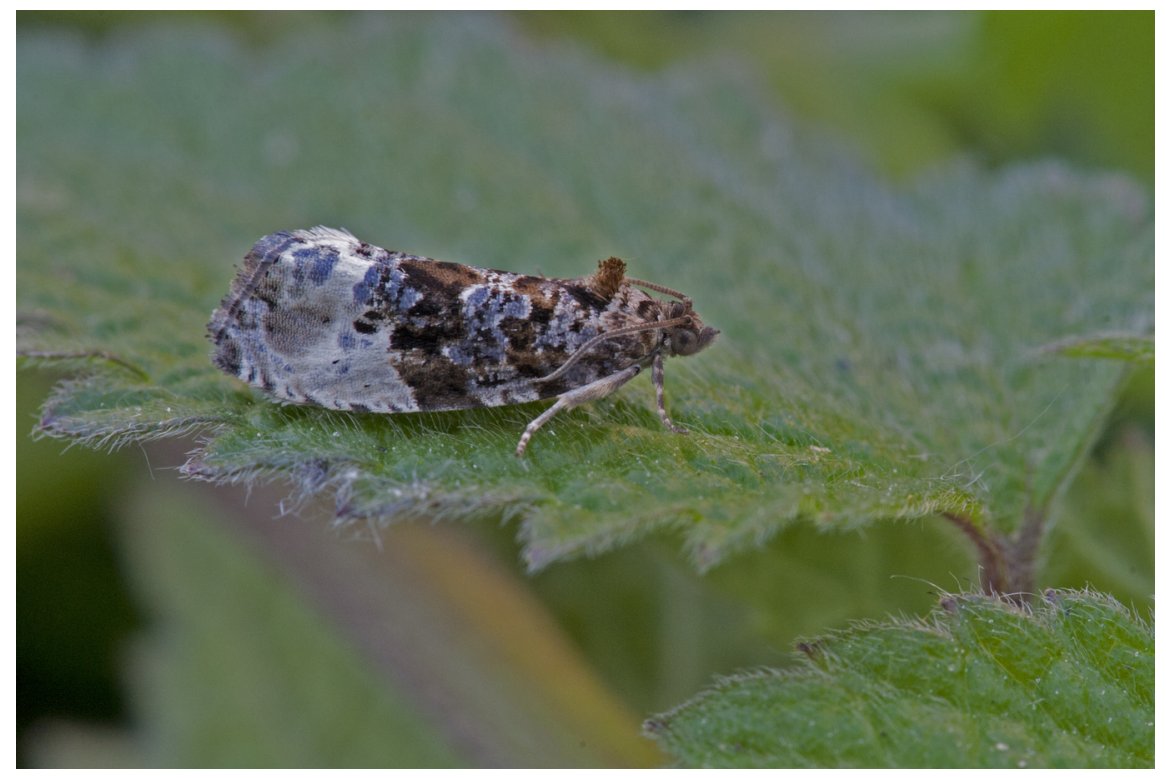

figures/gonzalez-figure2/gonzalez-figure2-eps-converted-to.pdf 
figures/gonzalez-figure3/gonzalez-figure3-eps-converted-to.pdf 